Published as: Nature. 2009 April 9; 458(7239): 757-761.

\title{
Role of Jhdm2a in regulating metabolic gene expression and obesity resistance
}

\author{
Keisuke Tateishi ${ }^{1,2, \dagger}$, Yuki Okada ${ }^{1,2}$, Eric M. Kallin ${ }^{1,2}$, and Yi Zhang ${ }^{1,2}$ \\ ${ }^{1}$ Howard Hughes Medical Institute, University of North Carolina at Chapel Hill, Chapel Hill, North \\ Carolina 27599-7295, USA \\ ${ }^{2}$ Department of Biochemistry and Biophysics, Lineberger Comprehensive Cancer Center, \\ University of North Carolina at Chapel Hill, Chapel Hill, North Carolina 27599-7295, USA
}

\section{Abstract}

\begin{abstract}
Recent studies indicate that the methylation state of histones can be dynamically regulated by histone methyltransferases and demethylases ${ }^{1,2}$. The H3K9-specific demethylase Jhdm2a (also known as Jmjd1a and $\mathrm{Kdm} 3 \mathrm{a}$ ) has an important role in nuclear hormone receptor-mediated gene activation and male germ cell development ${ }^{3,4}$. Through disruption of the Jhdm $2 a$ gene in mice, here we demonstrate that $\mathrm{Jhdm} 2 \mathrm{a}$ is critically important in regulating the expression of metabolic genes. The loss of Jhdm2a function results in obesity and hyperlipidemia in mice. We provide evidence that the loss of Jhdm2a function disrupts $\beta$-adrenergic-stimulated glycerol release and oxygen consumption in brown fat, and decreases fat oxidation and glycerol release in skeletal muscles. We show that Jhdm2a expression is induced by $\beta$-adrenergic stimulation, and that Jhdm 2 a directly regulates peroxisome proliferator-activated receptor $a$ (Ppara) and Ucpl expression. Furthermore, we demonstrate that $\beta$-adrenergic activation-induced binding of Jhdm $2 \mathrm{a}$ to the PPAR responsive element (PPRE) of the Ucpl gene not only decreases levels of H3K9me2 (dimethylation of lysine 9 of histone H3) at the PPRE, but also facilitates the recruitment of Ppar $\gamma$ and Rxra and their co-activators Pgc1aa(also known as Ppargc1a), CBP/ p300 (Crebbp) and Src1 (Ncoa1) to the PPRE. Our studies thus demonstrate an essential role for Jhdm2a in regulating metabolic gene expression and normal weight control in mice.
\end{abstract}

Using a hypomorphic Jhdm $2 a$ knockout model, we have previously shown an important role for the H3K9 demethylase Jhdm2a in spermatogenesis ${ }^{3}$. To further characterize the biological function of this demethylase, we created a new mouse line with the catalytic

CC2009 Macmillan Publishers Limited. All rights reserved

Correspondence and requests for materials should be addressed to Y.Z. (yi_zhang@med.unc.edu)..

†Present address: Department of Gastroenterology, University of Tokyo Hospital, Japan.

Author Contributions K.T. and Y.Z. designed the experiments and prepared the manuscript. K.T. performed most of the experiments. Y.O. provided the data for Supplementary Fig. 3. E.K. analysed microarray data and generated Supplementary Figs 6 and 7.

Author Information The primary microarray data is accessible from the NCBI Gene Expression Omnibus repository under the accession number GSE13552.

Supplementary Information is linked to the online version of the paper at www.nature.com/nature.

Figure S7. Jhdm2a deficiency affects PPAR signaling pathway in muscle.

Affymetrix microarray fold change data from RNA samples corresponding to Jhdm2a KO muscle vs. WT muscle was overlayed onto the PPAR signaling pathway using GenMAPP 2 software. Entities are colored by expression fold change in KO versus WT as indicated. 
jumonji $\mathrm{C}$ domain floxed by loxP sites (Supplementary Fig. 1a). Heterozygous mice were generated by crossing 3lox mice with EIIa-Cre transgenic mice (Supplementary Fig. 1a, b). Jhdm $2 a^{1 l o x / 1 l o x}$ (knockout) mice were obtained by intercrossing Jhdm $2 a^{1 l o x /+}$ mice, and were confirmed by PCR and western blot analysis (Supplementary Fig. 1b, c). Similar to the hypomorphic Jhdm $2 a$ mice, the complete knockout mice exhibit spermatogenesis defects (data not shown). Interestingly, the Jhdm $2 a$ knockout mice become obese in adulthood when compared to their wild-type littermates (Fig. 1a and Supplementary Fig. 2). The obese phenotype is also observed in Jhdm $2 a$ knockout mice backcrossed onto a C57BL/6 background (data not shown), as well as in the hypomorphic Jhdm $2 a$ mice on a mixed 129SV/Ola and C57BL/6 background (Supplementary Fig. 3), indicating that Jhdm2a deficiency leads to obesity irrespective of the genetic background. Consistent with a potential role for $J h d m 2 a$ in energy homeostasis, $J h d m 2 a$ is expressed at a relatively higher level in organs responsive to sympathetic nerve activity, such as brown adipose tissue (BAT) and skeletal muscle (Supplementary Fig. 4). In contrast, Jhdm2a expression in brain is almost undetectable (Supplementary Fig. 4).

Magnetic resonance imaging (MRI) analysis revealed marked body fat deposition in 4month-old knockout mice (Supplementary Fig. 2c). However, non-adipose tissues (lean) were comparable between wild-type and Jhdm $2 a$ knockout mice (Supplementary Fig. 2c). Similar to that observed in human obesity, large fat droplets are observed in white adipose tissue, as well as in muscle and liver (Fig. 1b). In addition, serum lipid content-including free fatty acid, triglyceride and total cholesterol—is significantly higher in the knockout mice (Fig. 1c). Furthermore, a high-fat diet accelerated the onset of obesity in the knockout mice (Fig. 1d). Collectively, these findings suggest that the loss of Jhdm2a function results in abnormal fat metabolism and obesity.

The body weight of an animal is maintained through a balance between food intake and energy expenditure ${ }^{5,6}$. To understand how $J h d m 2 a$ deficiency results in obesity, we first analysed food intake before the onset of obesity using 6-week-old mice, and found that caloric intake is not increased in the Jhdm2a knockout mice (Supplementary Fig. 5a). Similarly, the 16-week-old Jhdm2a knockout mice did not show an increased food intake, regardless of the fat content present in the diet (Supplementary Fig. 5b). In addition, the serum leptin level is not significantly altered in 6-week-old Jhdm $2 a$ knockout mice before they become obese (Supplementary Fig. 5c).

Given that the human counterpart of mouse Jhdm2a has been previously demonstrated to function as a transcriptional co-activator ${ }^{4}$, we compared the gene expression profiles of wild-type and $J h d m 2 a$ knockout skeletal muscles using Affymetrix microarray technology. We chose to perform the analysis in skeletal muscle because of the high expression of $J h d m 2 a$ and also its role in energy expenditure. Of the 43,000 probes analysed, 602 probes $(1.4 \%)$ were downregulated at least twofold and 220 probes $(0.05 \%)$ were upregulated at least twofold in Jhdm $2 a$ knockout soleus muscle. Gene ontology enrichment analysis indicated that the largest proportion of downregulated genes were involved in metabolic processes $(P=0.03)$ (Supplementary Fig. 6a), whereas genes involved in lipid metabolism were the most enriched $\left(P<1 \times 10^{-4}\right)$ (Supplementary Fig. 6b-e). Interestingly, pathway analysis showed that a significant proportion of the genes involved in PPAR signalling are 
downregulated in response to the $J h d m 2 a$ knockout $\left(P<1 \times 10^{-11}\right)$ (Supplementary Fig. 7). Quantitative PCR with reverse transcription (qRT-PCR) confirmed the downregulation of many genes involved in the PPAR pathway, including Ppara, Ucp2, MCAD (also known as Acadm), LCAD (Acadl), VLCAD (Acadvl) and Aqp7 but not Ppard (Fig. 2a). Consistent with the fact that multiple rate-limiting enzymes for fatty acid oxidation, such as MCAD, LCAD and VLCAD, were downregulated in the Jhdm $2 a$ knockout skeletal muscle, $\beta$-oxidation of palmitic acid was significantly decreased in primary myocytes derived from the Jhdm $2 a$ knockout newborn (Fig. 2b). Consistent with downregulation of Aqp7 (Fig. 2a), glycerol release in response to a $\beta$-adrenergic agonist is impaired in the $J h d m 2 a$ knockout soleus muscle (Fig. 2c). These results are consistent with previous demonstrations that Ppara has an essential involvement in fatty acid metabolism ${ }^{7-9}$. Notably, the results also indicate that the impaired expression of Ppara and its downstream target genes might be one of the causes of abnormal fat accumulation in the $J h d m 2 a$ knockout mice, because previous studies have shown that Ppara-deficient mice are prone to diet-induced obesity ${ }^{9}$ and that Aqp7 deficiency causes obesity in adult mice ${ }^{10}$.

To understand how the loss of Jhdm2a function results in the downregulation of Ppara and its target genes, we analysed the presence of Jhdm2a around defined PPRE sequences using chromatin immunoprecipitation (ChIP) ${ }^{11-14}$. Results indicate that Jhdm2a binds to the PPRE of Ppara, but not to the PPREs of MCAD, Acoxl or Scp2 (Fig. 2d). Consistent with the function of Jhdm2a as an $\mathrm{H} 3 \mathrm{~K} 9 \mathrm{me} 2$ demethylase, the level of $\mathrm{H} 3 \mathrm{~K} 9 \mathrm{me} 2$ at the region encompassing the PPRE of the Ppara gene is significantly increased in the Jhdm2a knockout muscle cells (Fig. 2e). To demonstrate that the reduced Ppara expression in $J h d m 2 a$ knockout muscle cells is a cell-intrinsic effect, we performed qRT-PCR and ChIP analysis using in vitro cultured primary myocytes derived from newborn wild-type and knockout mice. Results shown in Fig. 2f demonstrate that Ppara is decreased by 50\% in $J h d m 2 a$ knockout myocytes. ChIP analysis showed increased levels of H3K9me2 at the Ppara PPRE of Jhdm $2 a$ knockout myocytes (Fig. 2g). Notably, both Ppara expression and promoter $\mathrm{H} 3 \mathrm{~K} 9 \mathrm{me} 2$ levels at PPRE can be partially rescued by overexpression of human JHDM2A (Fig. 2f, g). These data collectively support the notion that Ppara is a direct target of Jhdm2a and that Jhdm2a plays an important part in lipid metabolism in skeletal muscle cells.

In addition to skeletal muscle, Jhdm2a is also highly expressed in BAT (Supplementary Fig. 4). Jhdm $2 a$ deficiency resulted in the enlargement and accumulation of lipid droplets in the BAT (Supplementary Fig. 8). Consistent with the BAT abnormities, Jhdm $2 a$ knockout mice showed defective adaptive thermogenesis (Fig. 3a). The phenotypic similarity between $J h d m 2 a$ knockout mice and mice lacking $\beta$-adrenergic receptors ${ }^{15}$ raises the possibility that Jhdm2a might be a critical factor in $\beta$-adrenergic signalling. Consistent with this notion, $\beta$ adrenergic-stimulated oxygen consumption and glycerol release are greatly reduced in the $J h d m 2 a$ knockout BAT (Fig. 3b, c). In addition, genes involved in mitochondrial functions including Ppara, Ucp 3, Cpt2 and $L C A D$ are also decreased in the Jhdm $2 a$ knockout BAT (Fig. 3d). Furthermore, analysis of the expression of Ucpl and Dio2, two key genes involved in thermogenesis in $\mathrm{BAT}^{16}$, demonstrated that cold-induced $U c p 1$ upregulation is almost completely blocked although Dio2 induction is not affected (Fig. 3e). Given the 
critical function of Ucp1 in cold sensitivity ${ }^{17}$, defective activation of $U c p l$ by $\beta$-adrenergic signalling is probably one contributing factor of defective thermogenesis in the $J h d m 2 a$ knockout BAT.

As one of the most important molecules involved in cold-induced thermogenesis in brown fat, the transcriptional regulation of $U c p 1$ has been extensively characterized ${ }^{16}$. In addition to Ppara and Ppar $\gamma$, other transcription factors and co-activators known to be involved in Ucpl activation include Rxra, Atf2, p300, Src1 and Pgc1a. qRT- PCR demonstrates that the expression of these genes is not significantly altered in the Jhdm2a knockout BAT (Supplementary Fig. 9), however cold-induced upregulation of Ppara is defective in knockout BAT (Supplementary Fig. 9). This indicates that the defect in cold-induced Ucpl induction in $J h d m 2 a$ knockout BAT might be mediated through Ppara, which is a direct target of Jhdm2a in muscle cells (Fig. 2d). Given that cold-induced Ucpl upregulation is intact in the Ppara deficient mice ${ }^{18}$, we explored the possibility that Jhdm2a directly regulates $U c p 1$ expression in response to cold exposure.

To ascertain that the effect of Jhdm2a on Ucpl expression is cell intrinsic, we performed short-hairpin RNA (shRNA)-mediated $J h d m 2 a$ knockdown in the brown adipose cell line HIB1B using lentivirus-based shRNA. Although Jhdm2a knockdown does not affect HIB1B differentiation (data not shown), the differentiated knockdown cells have impaired Ucp1 activation by isoproterenol (ISO), a general $\beta$-adrenergic receptor agonist (Fig. 4a), consistent with the result obtained in Jhdm $2 a$ knockout BAT (Fig. 3e). Importantly, enforced overexpression of human $J H D M 2 A$ in the knockdown cells partially rescued Ucp1 expression (Fig. 4b). Interestingly, both the messenger RNA and protein levels of Jhdm2a are upregulated in response to $\beta$-adrenergic receptor activation (Fig. 4c), supporting Jhdm2a as an integral component of the $\beta$-adrenergic signalling pathway. Similar to that observed in mouse brown fat tissues, the expression of the transcription factors and co-activators involved in Ucpl activation is not significantly altered in the HIB1B knockdown cells (Supplementary Fig. 10a). ISO-induced upregulation of Pgc1a is also maintained in HIB1B knockdown cells (Supplementary Fig. 10a). We next explored the possibility that Jhdm2a directly regulates $U c p 1$ expression. ChIP analysis demonstrated that Jhdm2a can bind to the $U c p 1$ enhancer region ${ }^{19}$ in a $\beta$-adrenergic receptor ligand-dependent manner. The binding is site-specific as Jhdm2a was not detected within the Ucpl coding region (Fig. 4d, amplicon B). As expected, the $\mathrm{H} 3 \mathrm{~K} 9 \mathrm{me} 2$ levels at the Jhdm2a binding site are specifically decreased in response to ISO treatment and this effect is abolished in the Jhdm2a knockdown cells (Fig. 4e). Notably, enforced overexpression of human JHDM2A in the knockdown cells partially rescued the increased $\mathrm{H} 3 \mathrm{~K} 9 \mathrm{me} 2$ level in an ISO-dependent manner (Fig. 4e). Collectively, these results indicate that Jhdm $2 a$ expression is regulated by the $\beta$-adrenergic signalling pathway and that Jhdm2a contributes to Ucpl activation by serving as a coactivator in response to $\beta$-adrenergic receptor activation.

Previous studies have indicated that Ppar $\gamma$ - and Rxra-mediated Ucpl activation requires the recruitment of co-activators ${ }^{20}$. In addition to removing the repressive $\mathrm{H} 3 \mathrm{~K} 9 \mathrm{me} 2$ mark at the Ucp 1 enhancer, Jhdm2a could contribute to Ucp 1 activation by affecting the recruitment of transcription factors and co-activators. To examine this possibility, we analysed the effect of the loss of Jhdm2a on the recruitment of transcription factors (Ppar $\gamma$, Rxra and Atf2) and 
co-activators (Pgc1a, CBP/p300 and Src1). We found that the binding of Ppary, Rxra and Atf 2 to the $U c p 1$ enhancer is increased in response to ISO treatment (Fig. 4f). However, theenhanced binding by Ppar $\gamma$ and Rxra disappeared in the Jhdm2a knockdown cells (Fig. 4f). Similar results are also observed for the co-activators (Fig. 4g). Given that Jhdm2a knockdown does not alter the protein levels of these transcription factors and co-activators (Supplementary Fig. 10b), the simplest explanation of the results is that Jhdm2a facilitates their recruitment in response to $\beta$-adrenergic stimulation. Our results, together with the previous demonstration that Ppar $\gamma$ recruitment to Ucpl enhancer is blocked in pCip (also known as Ncoa3) and $\operatorname{Srcl}$ double knockout $\mathrm{BAT}^{20}$, indicate that the binding of transcription factors and co-activators to the Ucpl enhancer affect one another. Collectively, our data suggest that Jhdm2a contributes to $\beta$-adrenergic-stimulated Ucp 1 activation by maintaining a low level of $\mathrm{H} 3 \mathrm{~K} 9 \mathrm{me} 2$ at the $U c p 1$ enhancer region, and by augmenting the recruitment of Ppary and Rxra and their co-activators to the Ucpl enhancer element.

In this study we identified Ppara and Ucpl, two of the important genes involved in controlling energy balance, as direct targets of Jhdm2a. Notably, the expression of these two genes, as well as $J h d m 2 a$, is induced after $\beta$-adrenergic stimulation ${ }^{16,21}$ (Figs $3 \mathrm{e}, 4 \mathrm{c}$ and Supplementary Fig. 9). Thus we propose that Jhdm2a mediates $\beta$-adrenergic signalling on the basis of the systemic energy demand. Consistent with this notion, Jhdm $2 a$ knockout mice and mice lacking $\beta$-adrenergic receptors have similar phenotypes that include defects in BAT function, cold intolerance, decreased oxygen consumption (Supplementary Fig. 11) and obesity without hyperphagia. Although the Jhdm2a deficiency does not affect metabolic hormone levels (Supplementary Fig. 12), the obesity phenotype supports an important role for Jhdm2a in regulating systemic metabolic control including Ppara and $\beta$-adrenergic signalling pathways. Although the entire mechanism underlying the phenotype reported here remains elusive, it is interesting to note that this is a unique mouse model in which a single epigenetic factor deficiency results in obesity.

\section{METHODS SUMMARY}

\section{Animal experiments}

All animal experiments were performed according to procedures approved by the Institutional Animal Care and Use Committee.

\section{Cell culture, viral infection and differentiation}

Primary myoblasts were established from neonatal mice as reported ${ }^{22}$. To generate a $J h d m 2 a$ knockdown HIB1B cell line, undifferentiated cells were infected with a lentiviral virus expressing an shRNA for $J h d m 2 a$ or control. The shRNA sequence for Jhdm $2 a$ was 5'-GCAGGTGTCACTAGCCTTAAT-3'. The differentiation of HIB1B cells was performed as previously described ${ }^{23}$. For the overexpression of the human JHDM2A gene, cells were infected with a retroviral vector expressing Flag- JHDM2A or a control before the cells were subjected to differentiation. 
Statistics

All results are presented as the mean and standard error. Statistical comparisons were by Student's $t$-tests. Statistical significance was set at $P<0.05$.

\section{Supplementary Material}

Refer to Web version on PubMed Central for supplementary material.

\section{Acknowledgments}

We thank B. M. Spiegelman for the HIB1B cells, L. Xia for construction of the targeting vector, K. E. Gardner for critical reading of the manuscript, D. Pump and K. Hua (UNC Clinical Nutrition Research Unit, DK56350) for calorimetry and MRI, and N. Takahashi for helpful comments. Y.Z. is an investigator of the Howard Hughes Medical Institute.

\section{References}

1. Klose RJ, Zhang Y. Regulation of histone methylation by demethylimination and demethylation. Nature Rev. Mol. Cell Biol. 2007; 8:307-318. [PubMed: 17342184]

2. Martin C, Zhang Y. The diverse functions of histone lysine methylation. Nature Rev. Mol. Cell Biol. 2005; 6:838-849. [PubMed: 16261189]

3. Okada Y, Scott G, Ray MK, Mishina Y, Zhang Y. Histone demethylase JHDM2A is critical for Tnp1 and Prm1 transcription and spermatogenesis. Nature. 2007; 450:119-123. [PubMed: 17943087]

4. Yamane K, et al. JHDM2A, a JmjC-containing H3K9 demethylase, facilitates transcription activation by androgen receptor. Cell. 2006; 125:483-495. [PubMed: 16603237]

5. Spiegelman BM, Flier JS. Obesity and the regulation of energy balance. Cell. 2001; 104:531-543. [PubMed: 11239410]

6. Evans RM, Barish GD, Wang YX. PPARs and the complex journey to obesity. Nature Med. 2004; 10:355-361. [PubMed: 15057233]

7. Reddy JK, Hashimoto T. Peroxisomal $\beta$-oxidation and peroxisome proliferator-activated receptor alpha: an adaptive metabolic system. Annu. Rev. Nutr. 2001; 21:193-230. [PubMed: 11375435]

8. Bedu E, Desplanches D, Pequignot J, Bordier B, Desvergne B. Double gene deletion reveals the lack of cooperation between PPARa and PPAR $\beta$ in skeletal muscle. Biochem. Biophys. Res. Commun. 2007; 357:877-881. [PubMed: 17466944]

9. Finck BN, et al. A potential link between muscle peroxisome proliferator-activated receptor- $\mathrm{a}$ signaling and obesity-related diabetes. Cell Metab. 2005; 1:133-144. [PubMed: 16054054]

10. Hibuse T, et al. Aquaporin 7 deficiency is associated with development of obesity through activation of adipose glycerol kinase. Proc. Natl Acad. Sci. USA. 2005; 102:10993-10998. [PubMed: 16009937]

11. Gulick T, Cresci S, Caira T, Moore DD, Kelly DP. The peroxisome proliferator-activated receptor regulates mitochondrial fatty acid oxidative enzyme gene expression. Proc. Natl Acad. Sci. USA. 1994; 91:11012-11016. [PubMed: 7971999]

12. Lopez D, Irby RB, McLean MP. Peroxisome proliferator-activated receptor a induces rat sterol carrier protein $\times$ promoter activity through two peroxisome proliferator-response elements. Mol. Cell. Endocrinol. 2003; 205:169-184. [PubMed: 12890579]

13. Pineda Torra I, Jamshidi Y, Flavell DM, Fruchart JC, Staels B. Characterization of the human PPARa promoter: identification of a functional nuclear receptor response element. Mol. Endocrinol. 2002; 16:1013-1028. [PubMed: 11981036]

14. Tugwood JD, et al. The mouse peroxisome proliferator activated receptor recognizes a response element in the $5^{\prime}$ flanking sequence of the rat acyl CoA oxidase gene. EMBO J. 1992; 11:433-439. [PubMed: 1537328] 
15. Bachman ES, et al. $\beta A R$ signaling required for diet-induced thermogenesis and obesity resistance. Science. 2002; 297:843-845. [PubMed: 12161655]

16. Lowell BB, Spiegelman BM. Towards a molecular understanding of adaptive thermogenesis. Nature. 2000; 404:652-660. [PubMed: 10766252]

17. Enerback $\mathrm{S}$, et al. Mice lacking mitochondrial uncoupling protein are cold-sensitive but not obese. Nature. 1997; 387:90-94. [PubMed: 9139827]

18. Kersten S, et al. Peroxisome proliferator-activated receptor a mediates the adaptive response to fasting. J. Clin. Invest. 1999; 103:1489-1498. [PubMed: 10359558]

19. Cassard-Doulcier AM, et al. Tissue-specific and $\beta$-adrenergic regulation of the mitochondrial uncoupling protein gene: control by cis-acting elements in the $5^{\prime}$-flanking region. Mol. Endocrinol. 1993; 7:497-506. [PubMed: 8388995]

20. Wang Z, et al. Critical roles of the p160 transcriptional coactivators p/CIP and SRC-1 in energy balance. Cell Metab. 2006; 3:111-122. [PubMed: 16459312]

21. Lomax MA, et al. Ontogenic loss of brown adipose tissue sensitivity to beta-adrenergic stimulation in the ovine. Endocrinology. 2007; 148:461-468. [PubMed: 17023522]

22. Rando TA, Blau HM. Primary mouse myoblast purification, characterization, and transplantation for cell-mediated gene therapy. J. Cell Biol. 1994; 125:1275-1287. [PubMed: 8207057]

23. Ross SR, et al. Hibernoma formation in transgenic mice and isolation of a brown adipocyte cell line expressing the uncoupling protein gene. Proc. Natl Acad. Sci. USA. 1992; 89:7561-7565. [PubMed: 1323843] 
a
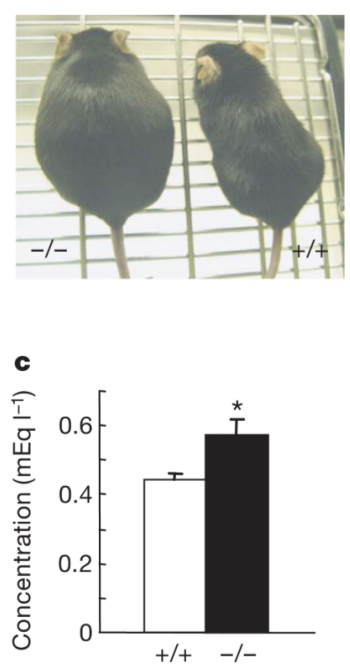

FFA

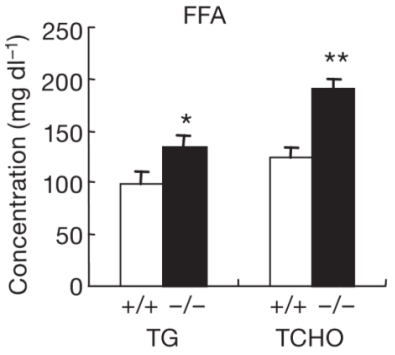

b
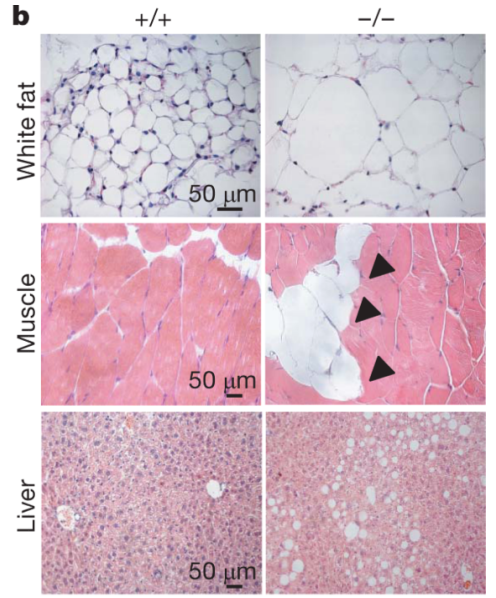

d

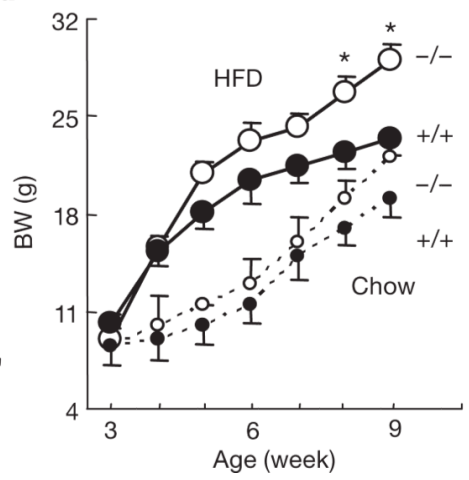

Figure 1. Jhdm2a-deficient mice exhibit obesity phenotypes

a, Jhdm $2 a$ knockout mice exhibit an obesity phenotype. Shown is a representative photograph of 7-month-old littermates. $\mathbf{b}$, Abnormal fat accumulation in organs of $J h d m 2 a^{-l-}$ mice. Haematoxylin and eosin (H\&E) staining of white adipose, muscle and liver derived from 7-month-old littermates. Arrowheads indicate the intramuscle fat deposition. Scale bars, $50 \mu \mathrm{m}$. c, Jhdm $2 a$ deficiency results in hyperlipidemia as indicated by the increased levels of serum free fatty acid (FFA), triglyceride (TG) and total cholesterol (TCHO) in $J h d m 2 a^{-/-}(n=16)$ compared with $J h d m 2 a^{+/+}(n=10)$ mice. $* P<0.05 ; * * P<$ 0.01. d, Growth curve of littermates fed with a high-fat diet (HFD) or normal chow. Solid lines, high-fat diet group; dotted lines, normal chow group. BW, body weight. $n=4$ per group; ${ }^{*} P<0.03$. Data are mean and s.e.m. 

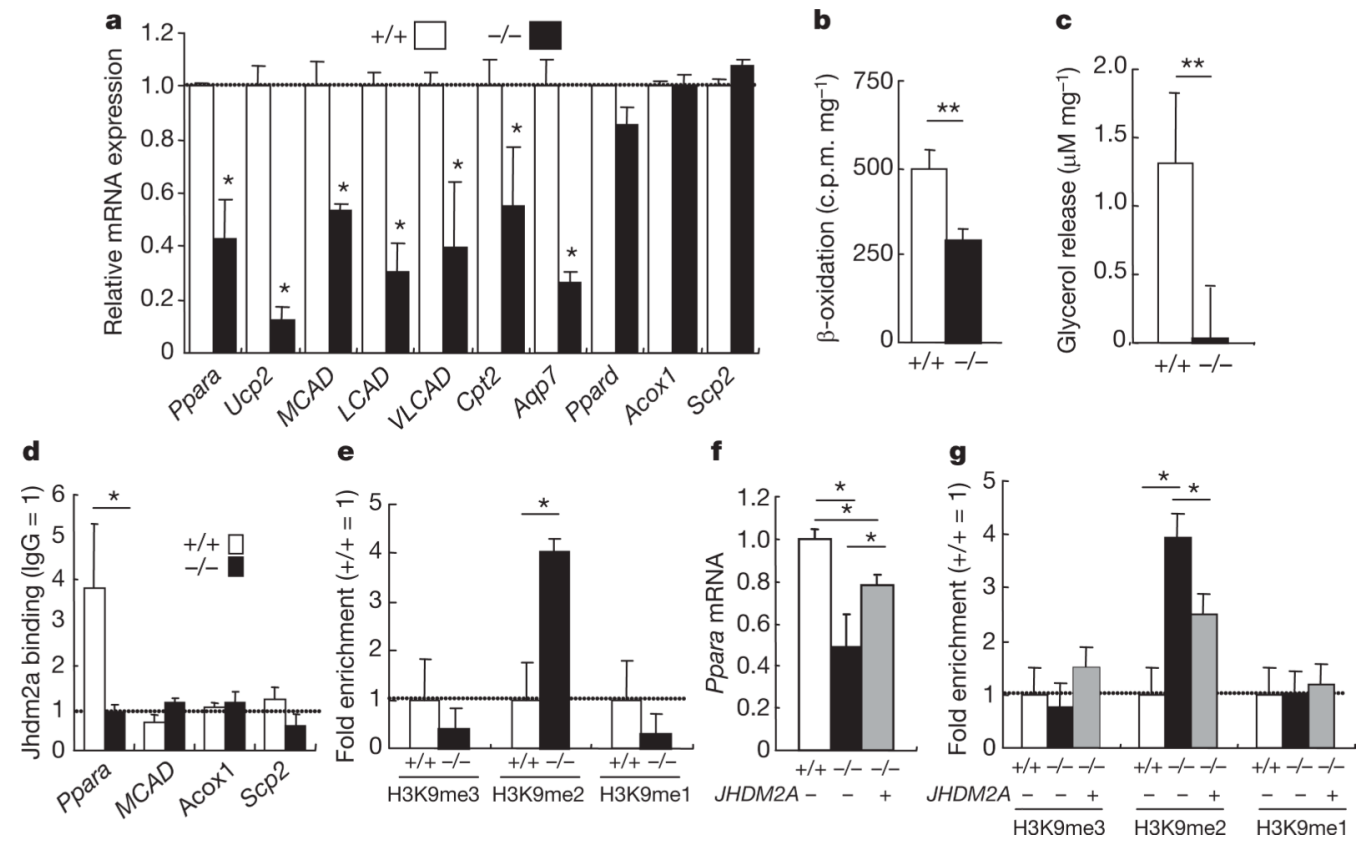

Figure 2. Jhdm2a deficiency affects the expression of metabolic genes and impairs $\beta$-oxidation and glycerol release in skeletal muscle

a, qRT-PCR analysis confirms the decreased expression of genes that are involved in PPAR signalling in the Jhdm $2 a$ knockout soleus muscles. $* P<0.05$. b, Jhdm $2 a$ deficiency results in decreased $\beta$-oxidation in primary muscle cells. $n=5$ per group; ${ }^{*} P<0.01$. c, $J h d m 2 a$ deficiency results in decreased glycerol release in isolated soleus muscle. $n=5$ per group; $* * P<0.01$. d, Jhdm2a directly binds to the PPRE of the Ppara enhancer. Soleus muscles were used for ChIP followed by qRT-PCR. Results are normalized to IgG. $* P<0.05$. e, $J h d m 2 a$ deficiency results in increased H3K9me2 levels within the Ppara enhancer. The signal in wild-type cells is set as $1 . * P<0.05$. f, Cultured Jhdm2a knockout myocytes show decreased Ppara expression, which can be partially restored by overexpression of the $J H D M 2 A$ gene. Wild-type primary cultured myocytes served as a control for qRT-PCR. $* P$ $<0.05$. g, ChIP analysis in the primary cultured myocytes. Jhdm2a deficiency causes the increase of the H3K9me2 level at the PPRE of the Ppara enhancer. This change can be partially rescued by overexpression of $J h d m 2 a$. The signal in wild-type cells is set as $1 . * P<$ 0.05. Data are mean and s.e.m. 

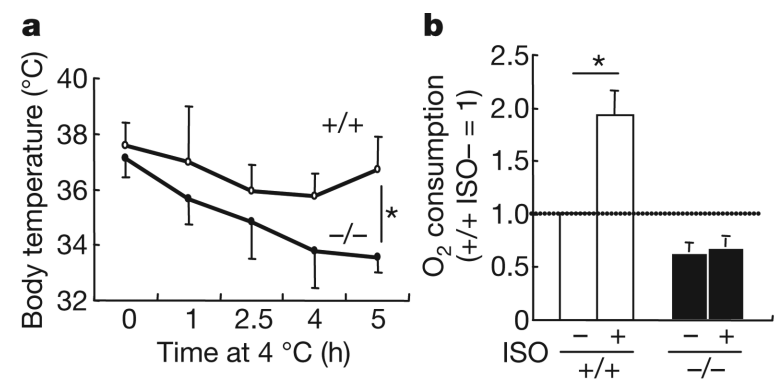

C

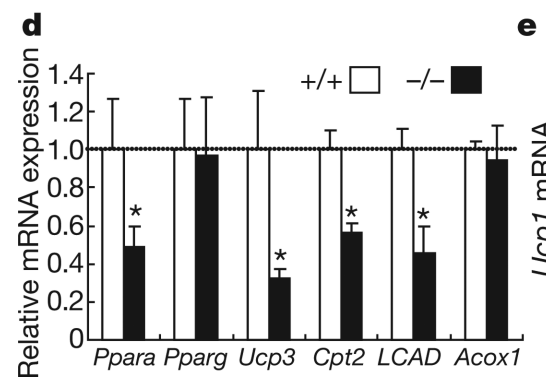

e

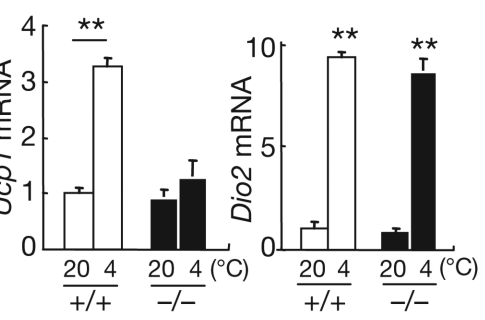

Figure 3. Jhdm2a deficiency results in functional defects in BAT

a, Jhdm2a deficiency impairs the ability of mice to maintain body temperature when exposed to cold. Shown is the body temperature of 12-week-old mice at different times after cold exposure $\left(4^{\circ} \mathrm{C}\right) . n=5$ per group; $* P<0.05$. b, Jhdm $2 a$ deficiency impairs ISO-induced oxygen consumption in BAT. $* P<0.05$. c,$J h d m 2 a$ deficiency results in decreased glycerol release in BAT. $* P<0.05$. d, qRT-PCR analysis demonstrates decreased expression of genes involved in mitochondrial function in the $J h d m 2 a$ knockout BAT. $* P<0.05$. $e$, $J h d m 2 a$ deficiency impairs cold-induced activation of $U c p 1$, but not Dio2, in BAT. $n>4$ per group. The mRNA level is normalized by $36 \mathrm{~B} 4 \mathrm{mRNA}$, and the relative quantity in $J h d m 2 a^{+/+}$BAT at $20{ }^{\circ} \mathrm{C}$ is defined as $1 . * * P<0.01$. Data are mean and s.e.m. 

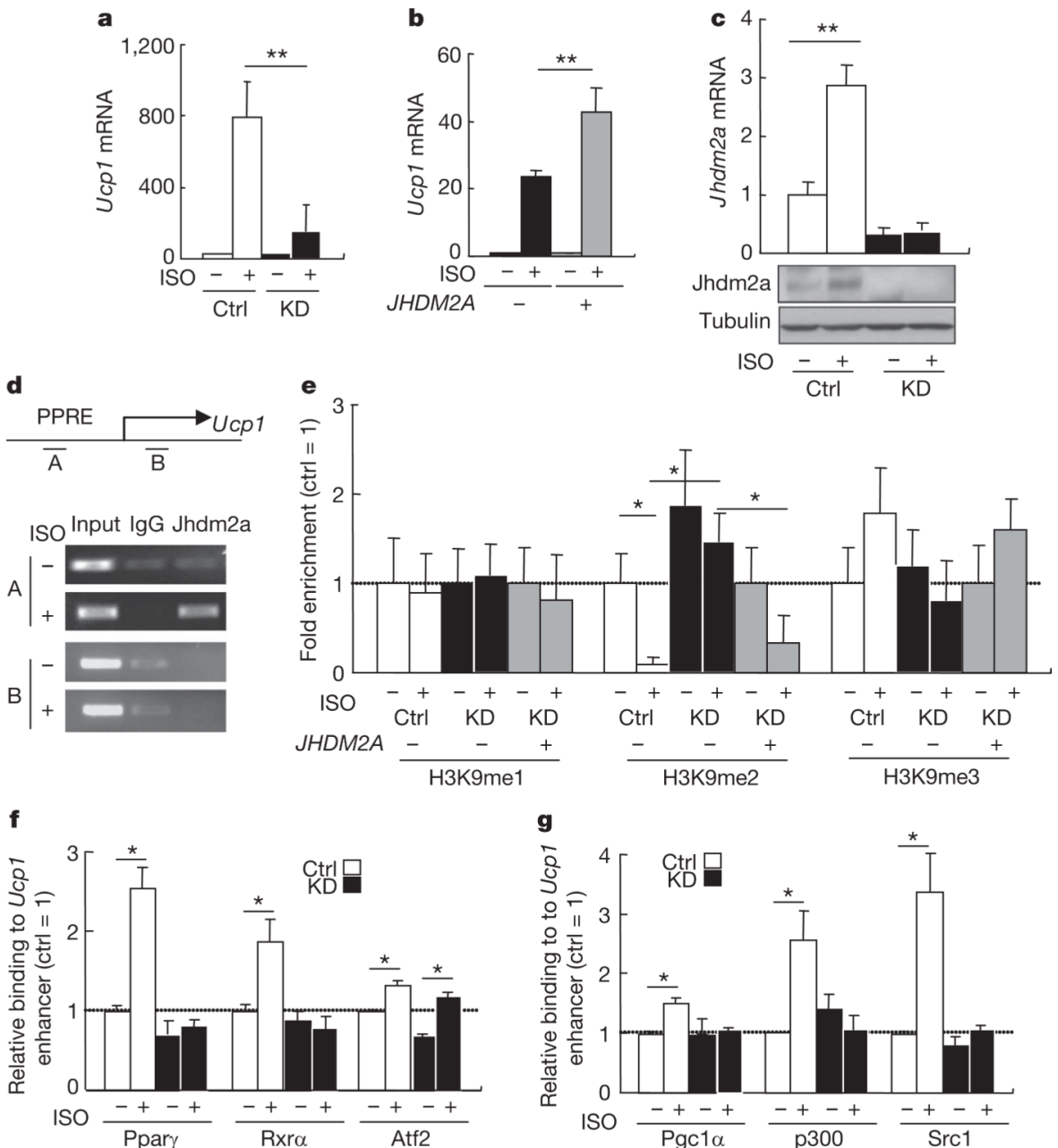

Figure 4. Jhdm2a is induced by $\beta$-adrenergic receptor activation and functions as a co-activator of $U$ cp 1

a, Jhdm $2 a$ knockdown (KD) impairs ISO-induced Ucpl transcriptional activation. Ctrl, control. ${ }^{* *} P<0.01$. b, Defective ISO-induced Ucpl activation caused by Jhdm $2 a$ knockdown can be partially restored by $J H D M 2 A$ overexpression $* P<0.05$. c, qRT-PCR (top panel) and western blot analysis (bottom panels) demonstrate that Jhdm2a is upregulated by ISO in HIB1B cells. $* * P<0.01$. d, ChIP analysis demonstrates that Jhdm2a directly binds to PPRE (amplicon A) of the Ucpl enhancer after $\beta$-adrenergic stimulation. e, $\mathrm{ChIP}$ analysis demonstrates that $\mathrm{H} 3 \mathrm{~K} 9 \mathrm{me} 2$ at the $U c p 1$ gene enhancer is demethylated by Jhdm $2 \mathrm{a}$ after ISO treatment. The Jhdm $2 a$ knockdown-induced increase in H3K9me2 level can be partially rescued by overexpression of $J H D M 2 A$ coupled with ISO treatment. *P < 0.05. f, ChIP followed by qPCR indicates Jhdm $2 a$ knockdown impairs $\beta$-adrenergic receptor activation-stimulated Ppar $\gamma$ and Rxra recruitment to the PPRE of the Ucpl gene enhancer. Results are normalized to $\mathrm{IgG}$, and are shown as the fold-enrichment relative to that in the control cells without ISO. $* P<0.05$. g, ChIP followed by qPCR indicates Jhdm2a knockdown impairs $\beta$-adrenergic receptor activation-stimulated recruitment of Ppar $\gamma$ and Rxra co-activators Pgc1a, p300 and Src1 to the PPRE of the Ucpl gene enhancer. Results 
are normalized to $\mathrm{IgG}$, and are shown as fold of enrichment relative to that in the control cells without ISO. $* P<0.05$. Data are mean and s.e.m. 